\title{
A new projection method for biological semantic map generation
}

\author{
Hoan N. Nguyen, Nicolas Wicker, David Kieffer, Olivier Poch
}

Laboratoire de bioinformatique et génomique intégratives, Institut de Génétique et de Biologie Moléculaire et Cellulaire, France University of Strasbourg, Illkirch Cedex, France.

Email: nguyen@igbmc.fr

Received 4 September 2009; revised 25 October 2009; accepted 26 October 2009.

\begin{abstract}
Low-dimensional representation is a convenient method of obtaining a synthetic view of complex datasets and has been used in various domains for a long time. When the representation is related to words in a document, this kind of representation is also called a semantic map. The two most popular methods are self-organizing maps and generative topographic mapping. The second approach is statistically well-founded but far less computationally efficient than the first. On the other hand, a drawback of self-organizing maps is that they do not project all points, but only map nodes. This paper presents a method of obtaining the projections for all data points complementary to the self-organizing map nodes. The idea is to project points so that their initial distances to some cluster centers are as conserved as possible. The method is tested on an oil flow dataset and then applied to a large protein sequence dataset described by keywords. It has been integrated into an interactive data browser for biological databases.
\end{abstract}

Keywords: Semantic Map; Dimension Reduction; Biological Database; SOM

\section{INTRODUCTION}

Thanks to the availability of the human and other genomes and the rapid progress of biotechnologies and information technologies, numerous large biomedical datasets have been generated. Modern biomedical information thus corresponds to a high volume of heterogeneous data that doubles in size every year and that covers very different data types, including phenotypic data, genotypic data as well as standards, processes, protocols or treatments used to generate information from raw data. In this context, systemic approaches are now needed to store, analyze and compare the huge amount of relevant information.

The first and second author contribute equally to this paper.
In addition, the knowledge provided by classical query services on biological data is often unsatisfactory (e.g. a list of proteins or sequences) and there is a need for user-friendly visual representations of the data. Such a representation exists and is called a feature or semantic map. It is used to visualize "land maps" in two or three dimensions that represent, for example, the distribution (similarity and neighborhood) of protein annotations in biological databases. When query results are represented on the map, the repartition of the proteins can be easily observed, as well as their proximity to clusters labeled according to their content. In addition, it is straight-forward to superpose the information obtained from additional requests. Thus, a semantic map can greatly facilitate the interpretation of results from large scale data analyses. To quote a few examples, semantic maps have already been used in fluid mechanics [1], astronomy [2], internet data mining [3,4], scientific literature mining [5] and biology [6].

Many low-dimensional methods have been devised $[5,7,8,9]$ and two of the most popular are the WEBSOM method [9] and the Generative Topographic Mapping (GTM) [1]. These two methods are briefly outlined below.

WEBSOM originates from self-organizing maps [10] which is a classification algorithm where nodes move towards cluster centers. In WEBSOM, the nodes are fixed on a two-dimensional grid and at the same time live in the space of the dataset, typically a $\mathrm{R}^{p}$ space. First, a point $y$ is picked at random from the dataset.

Next, the closest node $w_{i}$ in $\mathrm{R}^{p}$ is selected and then each node $w_{j}$ moves towards $\mathrm{y}$ according to the equation $\quad w_{j}(t+1)=w_{j}(t)+\eta(t) h_{i j}(t)\left\|y-w_{j}(t)\right\| \quad$ where $\eta(t)$ is the learning rate decreasing in time and $h_{i j}(t)$ is a neighborhood function in the two-dimensional grid. These steps are then iterated for all data points. The initialization of the p-dimensional space can be performed randomly, but a more effective method is to select points 
along the two first principal axes of the dataset [4]. Finally, the dataset is used again by assigning each point to its closest node in the p-dimensional space using a Euclidean distance. Then, for each node, the number of points it has captured is taken as its density up to a given scaling factor (the size of the dataset).

The generative topographic map (GTM) [1] is a statistical method which is provably (locally) convergent and which does not require a shrinking neighborhood or a decreasing step size. It is a generative model: the data is assumed to arise by probabilistically picking points in a low-dimensional space and mapping them to the observed high-dimensional input space. The statistical model can be described in the following way:

$$
p\left(y \mid x_{i}, W, \beta\right)=\left(\frac{\beta}{2 \pi}\right)^{p / 2} \exp \left\{-\frac{\beta}{2}\left\|W . \varphi\left(x_{i}\right)-y\right\|^{2}\right\}
$$

where $x_{i}$ is a two-dimensional grid node, $\beta$ is a scaling parameter, $W . \varphi\left(x_{i}\right)$ a generalized regression model, $W$ a $p \times m$ matrix and the elements of $\varphi(x)$ consist of $m$ basic functions $\varphi_{j}(x)$ typically equal to radially symmetric Gaussians centered on the nodes of a two-dimensional grid. The parameters $W$ and $\beta$ of the model are estimated through the expectation-maximization (EM) algorithm [11]. This model can be considered to be the probabilistic counterpart of SOM/WEBSOM. However, the WEBSOM method is quicker than GTM when large amounts of data must be dealt with, especially if the winner selection is optimized so that millions of documents and nodes can be treated [4].

An alternative choice is to follow Flexer's approach [12] which first clusters the points in the data space and then projects cluster centers using Sammon's multidimensional scaling method [13]. However this means that only a subset of points are effectively projected. In this paper, we present a complementary method that projects all points using their distances to the cluster centers.

First this new projection method is presented, then it is evaluated on a benchmark data set and compared to other methods. Finally, it is used in the results section to generate a semantic map in the context of a new integrative navigator for biological databases.

\section{METHODS}

The principle of the presented method is to project points after they have been clustered and the cluster centers have been projected onto a two-dimensional map. This is done by conserving as much as possible the original distances between the points and the cluster centers. Basically, for each point indexed by $i$, the two- dimensional coordinates are search such as to minimize the difference between the distances computed in the $n$-dimensional data space with those computed on the map.
This comes down to finding the point $x_{i}$ in two dimensions minimizing the following function $E\left(x_{i}\right)$ :

$$
E\left(x_{i}\right)=\sum_{g=1}^{G}\left(\sum_{k=1}^{2}\left(x_{k}-c_{g, k}\right)^{2}-d_{g}^{2}\right)^{2}
$$

with $d_{g}$ denoting the distance between point $i$ and cluster $g$ and $c_{g, k}$ the projection of the $k^{\text {th }}$ cluster center. The Newton-Raphson algorithm was used to minimize $E\left(x_{i}\right)$. At each step, $x_{i}^{t+1}=x_{i}^{t}-H^{-1} . \nabla E$ with $H$ the Hessian and $\nabla E$ the gradient of $E$.

$$
\nabla E=\left(\begin{array}{l}
\frac{\partial E}{\partial x_{1}} \\
\frac{\partial E}{\partial x_{2}}
\end{array}\right), H=\left(\begin{array}{ll}
\frac{\partial^{2} E}{\partial x_{1}^{2}} & \frac{\partial^{2} E}{\partial x_{1} \partial x_{2}} \\
\frac{\partial^{2} E}{\partial x_{1} \partial x_{2}} & \frac{\partial^{2} E}{\partial x_{2}^{2}}
\end{array}\right)
$$

The optimizing function is not convex as the Hessian is not always semi-definite positive. To show this, it is sufficient to find a point $X$ verifying $X^{\prime} H X<0$. In particular, we show that $H_{11}$ can be negative which is also sufficient. First let us note that

$$
\begin{gathered}
\frac{\partial E}{\partial x_{l}}=\sum_{g=1}^{G} 2\left(\sum_{k=1}^{2}\left(x_{k}-c_{g, k}\right)^{2}-d_{g}^{2}\right) 2\left(x_{l}-c_{g, l}\right) \\
\frac{\partial^{2} E}{\partial x_{l}^{2}}=8 \sum_{g=1}^{G}\left(x_{l}-c_{g, l}\right)^{2}+4 \sum_{g=1}^{G}\left(\sum_{k=1}^{2}\left(x_{k}-c_{g, k}\right)^{2}-d_{g}^{2}\right) \\
\text { and then set } \\
\left\{\begin{array}{l}
x_{1}=c_{1,1}=c_{2,1}=c_{3,1} \\
d_{1}=\sqrt{\left(x_{1}-c_{1,1}\right)^{2}+\left(x_{2}-c_{1,2}\right)^{2}} \\
d_{2}=\sqrt{\left(x_{1}-c_{2,1}\right)^{2}+\left(x_{2}-c_{2,2}\right)^{2}} \\
d_{3}>\sqrt{\left(x_{1}-c_{3,1}\right)^{2}+\left(x_{2}-c_{3,2}\right)^{2}}
\end{array}\right.
\end{gathered}
$$

Thus, $H_{11}=4\left(\left(x_{1}-c_{3,1}\right)^{2}+\left(x_{2}-c_{3,2}\right)^{2}-d_{3}^{2}\right)<0$

Consequently, a global optimization process was performed using different initial values. Each cluster center projection was used as an initial value and the best solution after convergence was kept.

\section{RESULTS AND DISCUSSION}

\subsection{Validation Using the Oil Flow Dataset}

To validate the new points projection method, a previously established oil flow dataset [14] was used as a benchmark. This training dataset is available at http://www.ncrg.aston.ac.uk/GTM/ and contains 1000 


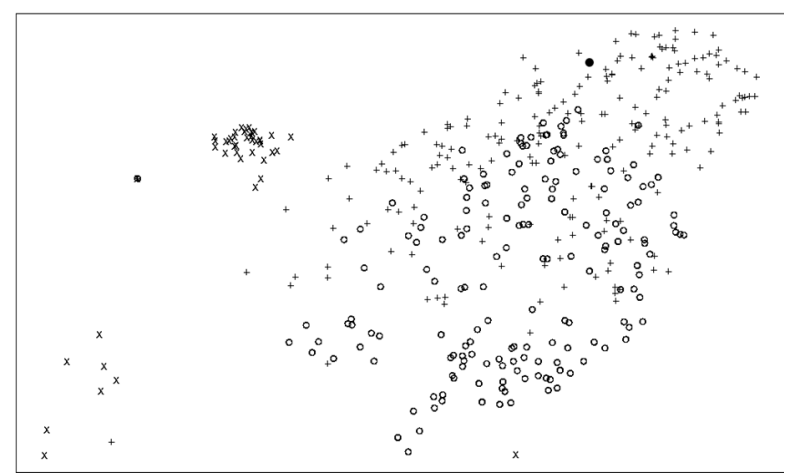

Figure 1. New projection of the dataset. Results of the presented projection on the oil flow dataset. Crosses, circles and plus-signs represent stratified, annular and homogeneous multi-phase configurations respectively. The three group separations are clearly identified.

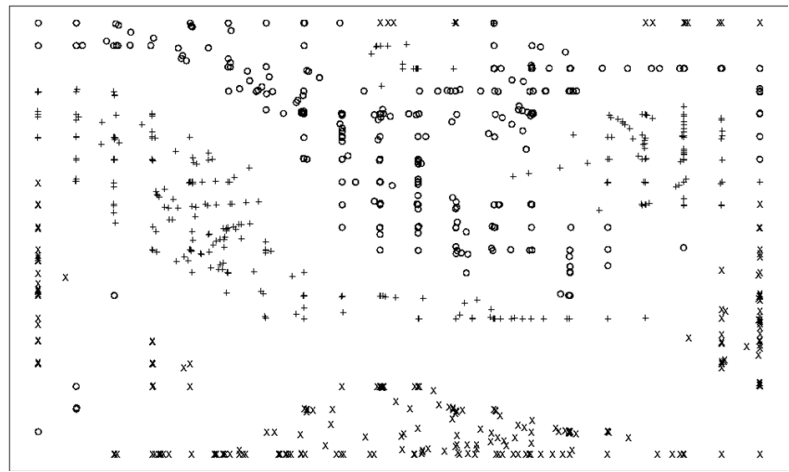

Figure 2. Oil flow dataset after GTM. After projection of the oil flow dataset using the Generative Topographic Mapping, the three group separations are clearly separated, but in a complex way that is far from linear.

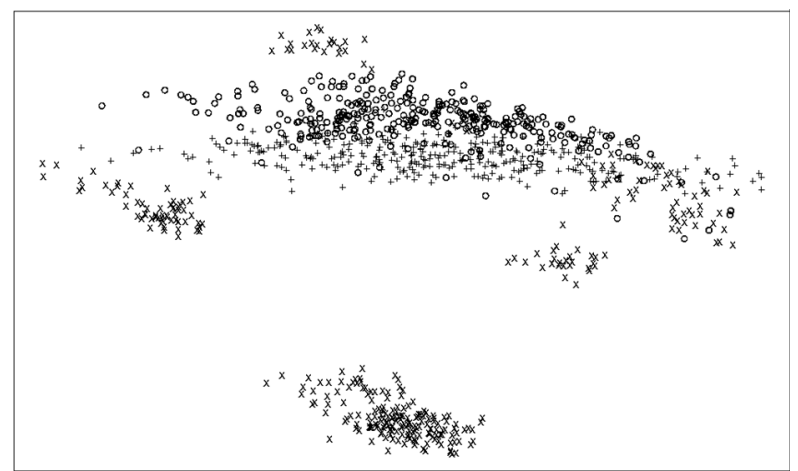

Figure 3. Oil flow dataset after PCA projection. After projection of the oil flow dataset using principal component analysis, the separation of the three groups is not clearly identified. In particular, the crosses are very scattered.

points in 12 dimensions corresponding to 12 measurements on the mixture of oil, water and gas passing through a pipeline. The three phases in the pipe can belong to three different configurations corresponding to laminar, homogeneous and annular flows.
First, the dataset was clustered into 15 clusters and the cluster centers projected according to Sammon's multidimensional scaling method [13]. Then the 1000 points were projected in two dimensions using the method described above. The results are shown on Figure 1, where it be seen that three different groups are rather well linearly separated. The groups obtained with the GTM and principal component analysis (PCA) methods are shown on Figures 2 and 3 respectively. In order to objectively measure the quality of these results, we computed the ratio of the between-class inertia and the total inertia for each method. For our method, GTM and the PCA, we obtained a ratio of $0.83,0.25$ and 0.23 respectively, thus confirming the visual impression. Nevertheless, it should be stated that, if only separation is desired and not specifically linear separation, GTM performs better, even though it has the drawback of making the underlying grid very visible.

\subsection{Semantic Map Generation for Biological Database}

The Laboratory of Genomics and Integrative Bioinformatics (LGBI) at the IGBMC Strasbourg, has developed a new high-performance biomedical information system, called the BIRD System [15,16]. BIRD is able to integrate very quickly heterogeneous data either from the large generalist databases (sequence, structure, function and evolution, etc.) or from specialized databases dedicated to high throughput biology (transcriptomics, interactomic, etc.) in a relational database (IBM DB2). Thus, it allows to organize massive sets of biomedical data according to real world requirements. An original biological query engine, called BIRDQL, has been designed to facilitate access to the heterogeneous databases and to allow pertinent information extraction via a web server. This system has been used in the Decrypthon computing grid [17] in order to provide data to the runtime applications.

To complete the visualization and analyze functionalities of the BIRD System, the new method described above to build semantic maps was integrated in the BIRD query engine (BIRDQL). The maps can be used to explore the data using a combination of high level queries and area selections (Figure 4). The method was tested by building a semantic map of the Uniprot database [18] using the keyword descriptions for each protein. After removal of redundant vectors, we obtained 60,000 vectors $z_{1}, \ldots z_{60000}$ in a 914-dimensional space corresponding to the 914 keywords extracted from about 6 million proteins. In the following lines, to avoid focusing on the numerical details, we will consider $n$ proteins described by $p$ keywords where $n$ and $p$ stand for 60000 and 914 respectively.

Before projecting the points, some preliminary steps were necessary: 


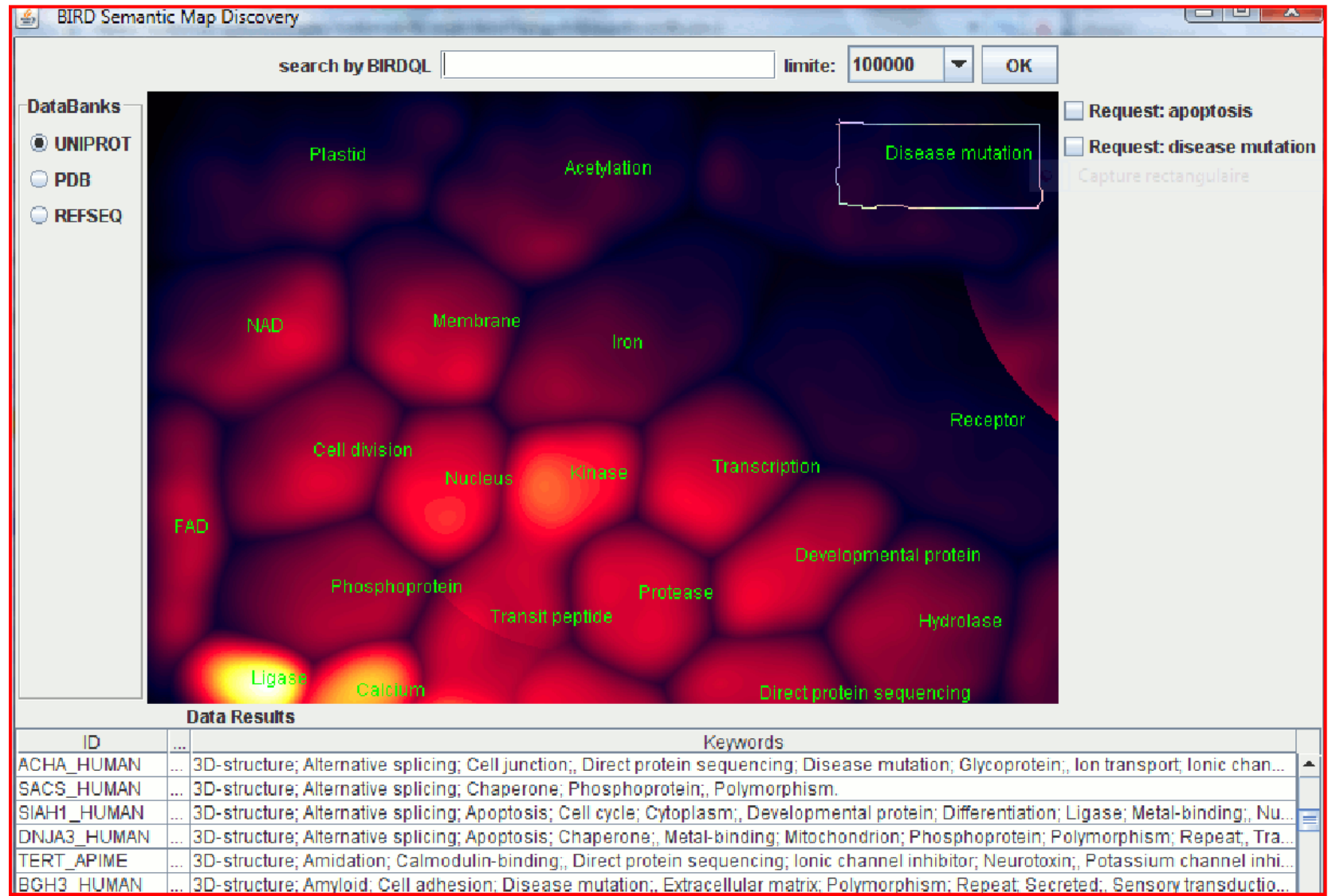

Figure 4. Semantic map with density colours and most frequent keyword labels.

\section{Step 1: dimension reduction}

The $n$ proteins were described by $p$ keywords and were thus represented by $n$ points $z_{1}, \ldots, z_{n}$ in $p$ dimensions. As in the preprocessing step of WEBSOM $[3,4]$, an initial dimension reduction was performed to reduce $p$ coordinates to $p^{*}$ using random projection directions. More specifically, random vectors $v_{j}$ were generated on the $p^{*}$-dimensional unit-sphere and then new coordinates were obtained by computing the scalar product $y_{i j}=<v_{j}, z_{i}>$ on each document $i$. Thus, the $n$ proteins were described by $n$ points $y_{1} \ldots y_{n}$.

\section{Step 2: mixture models clustering}

In a second step, these points were clustered using mixture models. Mixture models are a powerful method to cluster datasets of points described by coordinates. The points are assumed to be independent realizations from a mixture of several distributions. Here the mixture is only briefly described for $G$ components $f_{\alpha_{1}}, \ldots, f_{\alpha_{G}}$ with parameters $\alpha_{1} \ldots, \alpha_{G}$. A general presentation of this method and its applications can be found in $[19,20,21,22]$. If $\tau_{1}, \ldots, \tau_{G}$ indicate the different weights of the components, the likelihood of the model for $n$ points $y_{1}, \ldots, y_{n}$ is expressed as:

$$
L_{M}\left(\tau_{1}, \ldots, \tau_{G}, \alpha_{1}, \ldots \alpha_{G} \mid y_{1}, \ldots, y_{n}\right)=\prod_{i=1}^{n} \sum_{g=1}^{G} \tau_{g} f_{\alpha g}\left(y_{i}\right)
$$

The estimation of the different coefficients of the mixture model is commonly performed via the EM (Expectation-Maximization) algorithm of Dempster [11]. Here, in order to simplify the estimation, a variant of the EM algorithm called CEM was used [22]. In this application $G$ was chosen to be equal to 30 .

\section{Step 3: cluster centers projection}

Once $G$ clusters were obtained, the centers of gravity $c_{1}^{*}, \ldots, c_{G}^{*}$ were computed in the $\mathrm{p}$-dimensional space. Then, multidimensional scaling (MDS) [23] was applied on the cluster centers to produce two-dimensional coordinates $c_{1}, \ldots, c_{G}$. MDS was used because Sammon's method [13] failed on this dataset, since it produced many points with the same coordinates.

After these three preliminary steps, the points were projected on the map using the new projection method. The density $m(x)$ for each point $x$ of the map is given using a kernel method [24]: 


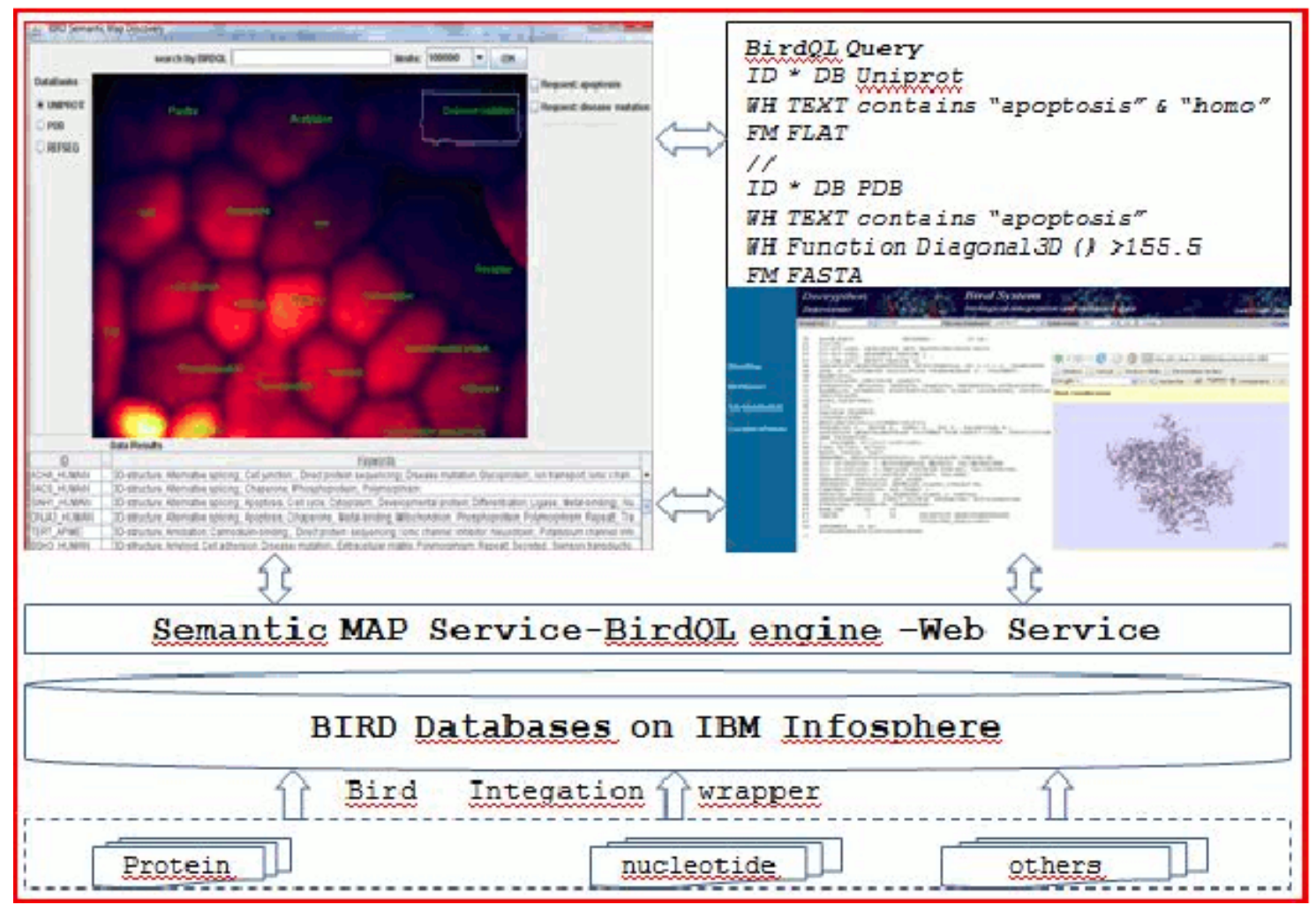

Figure 5. The global architecture of the Semantic Map Discovery prototype coupled with the BIRD System using the BirdQL query engine.

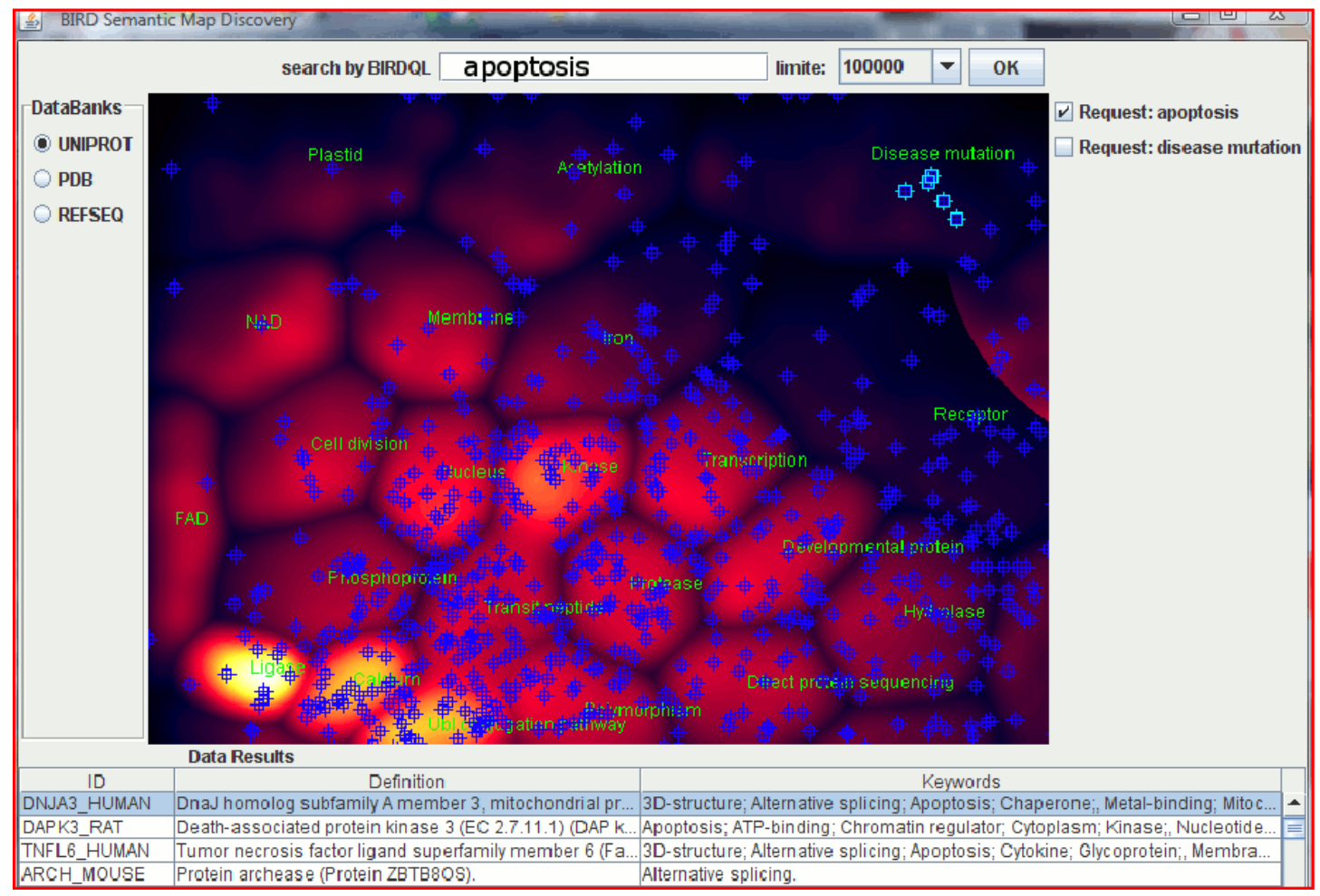

Figure 6. Semantic map with selected proteins. The labels represent the most frequent keywords present inside the cluster points which are not shared between different clusters. 


$$
m(x) \frac{1}{n} \sum_{i=1}^{n} \frac{1}{2 \pi\left|\sum\right|^{1 / 2}} \exp \left\{\left(x-x_{i}\right) \sum^{-1}\left(x-x_{i}\right)\right\}
$$

Then, a color scale ranging from purple to white, with intermediary colors red, orange and yellow was assigned to each point according to its density. The map is represented in Figure 4.

This visual representation allows a global comprehension of the whole database, which is easier to understand than numerical or textual data. Some important keywords shared by many proteins are visible on this map, such as kinase, ligase and protease. At the same time, frequent keywords, such as "complete proteome", that are non-informative, are avoided because they are shared by several clusters. Another observation is that the density is far from being homogeneous, the map being more crowded in the bottom-left corner than elsewhere.

When using the integrated biological query engine BIRD-QL of the BIRD System via a web service or http protocol, as shown in Figure 5, the selected proteins are represented on the maps by a plus sign of a given color. If different selections have been performed, different colors are used. An example is shown in Figure 6, where proteins selected by a query with the keyword "apoptosis" are shown by blue plus signs. Some of these proteins were selected by the user and are surrounded by a white square. One of the proteins, DNJA3, belongs to the small cluster labeled "disease mutation" but does not possess the "disease mutation" keyword. Interestingly its deficiency implies dilated cardiomyopathy [25] (MIM608382).

There is still room for improvement in the construction of semantic maps both at the algorithmic level and at the software functionality level. The point's projection is formalized as a global optimization problem and currently, it is resolved simply using different starting points with the Newton-Raphson method. However global optimization methods could also be tested [26,27]. From a practical point of view it would also be useful to determine how many clusters or nodes are necessary to achieve a good projection of the data points.

\section{CONCLUSIONS}

The main contribution of this work is a new computational solution to the construction of semantic maps. The idea is to project points by locating them according to cluster centers. This method can thus be coupled with other methods such as self-organizing maps or Flexer's approach.

\section{ACKNOWLEDGEMENTS}

This work was supported by the CNRS, the University of Strasbourg and the Décrypthon program initiated by the Association Française contre les Myopathies, IBM and the CNRS. We are grateful to all internship students who participated in this work by programming some parts of it, namely Xavier Brotel, Jérémy Némo Trouslard and Julien Cadet. The authors would like to thank Anne Friedrich, Laurent Philippe Albou and Julie Thompson for helpful suggestions.

\section{REFERENCES}

[1] Bishop, C.M., Svens'en, M. and Williams, C.K.I. (1998) GTM: the generative topographic mapping, Neural Computation, 10, 215-234.

[2] Lesteven, K. (1995) Multivariate data analysis applied to bibliographical information retrieval: SIMBAD quality control. Vistas in Astronomy, 39, 187-193

[3] Kaski, S. (1998) Dimensionality reduction by random mapping: Fast similarity computation for clustering, Proceedings of IJCNN'98, International Joint Conference on Neural Networks, IEEE Service Center, 413-418.

[4] Lagus, K., Kaski, S. and Kohonen, T. (2004) Mining massive document collections by the WEBSOM method. Information Sciences, 163, 135-156.

[5] Chen, C. (2005) CiteSpace II: Detecting and visualizing emerging trends and transient patterns in scientific literature. Journal of the American Society for Information Science, 57, 359-377.

[6] Grimmelstein, M. and Urfer, W.W. (2005) Analyzing protein data with the generative topographic mapping approach. innovations in classification, data science, and information systems, Baier, D. and Wernecke, K.D. Springer Berlin Heidelberg, 585-592.

[7] Ossorio, P.G, (1966) Classification space: a multivariate procedure for automated document indexing and retrieval. Multivariate Behavioral Research, 1, 479-524.

[8] Deerwester, S., Dumais, S.T., Furnas, G.W., Landauer, T. K. and Harshman R. (1990) Indexing by latent semantic indexing. Journal of the American Society for Information Science, 41, 391-407.

[9] Kohonen, T. (1997) Self-Organizating Maps, SpringerVerlag.

[10] Kohonen, T. (1982) Analysis of a simple self-organizing process. Biological Cybernetics, 44, 135-140.

[11] Dempster, A., Laird, N. and Rubin, D. (1977) Maximum likelihood from incomplete data via the $\{\mathrm{EM}\}$ algorithm. Journal of the Royal Statistical Society, Ser. B, 39, 249-282.

[12] Flexer, A. (1997) Limitations of self-organizing maps for vector quantization and multi-dimensional scaling. Advances in neural information processing systems, 9, 445-451.

[13] Sammon J.W. (1969) A non-linear mapping for data structure analysis. IEEE Transactions on Computers, 18, 401-409.

[14] Bishop, C.M. and James G.D. (1993) Analysis of multiphase flows using dual-energy gamma densitometry and neural networks. Nuclear Instruments and Methods in Physics Research, Section A, 327, 580-593.

[15] Nguyen, H., Berthommier, G., Friedrich, A., Poidevin, L., Ripp, R., Moulinier, L. and Poch, O. (2008) Introduction to the new Decrypthon Data Center for biomedical data, Proc CORIA', 32-44.

[16] BIRDQL-Wikili, http://alnitak.u-strasbg.fr/wikili/index.php/BIRDQL.

[17] Décrypthon: le grid-computing au service de la génomique et la protéomique. http://www.decrypthon.fr. 
[18] (2008) The uniProt consortium. The Universal Protein Resource (UniProt). Nucleic Acids Research, 36, D190D195.

[19] Titterington, D., Smith, A. and Makov, U. (1985) Statistical analysis of finite mixture distribution, John Wiley and Sons.

[20] McLachlan, G. and Basford, K. (1988) Mixture models: inference and applications to clustering, Marcel Dekker.

[21] Banfield, J. and Raftery, A. (1993) Model-based Gaussian and non-Gaussian clustering. Biometrics, 49, 803-821.

[22] Celeux, G. and Govaert, G. (1992) A classification EM algorithm for clustering and two stochastic versions, Journal of Computational Statistics and Data Analysis, 14, 315-332.

[23] Mardia, K.V., Kent, J.T. and Bibby, J.M. (1979) Multi- variate Analysis, Academic Press.

[24] Parzen, E., (1962) On estimation of a probability density function and mode. Annals of Mathematical Statistics, 33, 1065-1076.

[25] Hayashi, M., Imanaka-Yoshida, K., Yoshida, T., Wood, M., Fearns, C., Tatake, R. and Lee, J. (2006) A crucial role of mitochondrial Hsp40 in preventing dilated cardiomyopathy, Nature Medecine, 12, 128-132.

[26] Laguna, M. and Marti, R. (2005) Experimental testing of advanced scatter search designs for global optimization of multimodal functions, Journal of Global Optimization 33, 235-255.

[27] Neumaier, A., Shcherbina, O., Huyer, W. and Vinko, T. (2005) A comparison of complete global optimization solvers, Mathematical Programming, 103, 335-356. 